\title{
Urinary C-Peptide Creatinine Ratio as a Non-Invasive Tool for Identifying Latent Autoimmune Diabetes in Adults (LADA)
}

This article was published in the following Dove Press journal: Diabetes, Metabolic Syndrome and Obesity: Targets and Therapy

\author{
Wei Liu' \\ Xingquan Huang ${ }^{1,2}$ \\ Xiuying Zhang' \\ Xiaoling Cai (i)' \\ Xueyao Han' \\ Xianghai Zhou (D) ${ }^{1}$ \\ Ling Chen' \\ Rui Zhang' \\ Siqian Gong' \\ Yanai Wang $\mathbb{D}^{\prime}$ \\ Linong Ji'
}

'Department of Endocrinology and Metabolism, Peking University People's Hospital, Beijing, People's Republic of China; ${ }^{2}$ Department of Endocrinology and Metabolism, The Second Affiliated Hospital of Hebei North University, Zhangjiakou, Hebei, People's Republic of China
Correspondence: Linong Ji

Department of Endocrinology and Metabolism, Peking University People's Hospital, No. II, Xi Zhi Men Nan Street, Beijing 100044, People's Republic of China Tel +86- |0-88324108

Fax +86-10-8832437|

Email jiln@bjmu.edu.cn
Purpose: Latent autoimmune diabetes in adults (LADA) is a slowly progressing form of immune-mediated diabetes that combines phenotypical features of both type 2 diabetes mellitus (T2DM) and type 1 diabetes mellitus (T1DM), meaning that accurate and early diagnosis of this subtype of diabetes is critical for optimal long-term management. Urinary C-peptide creatinine ratio (UCPCR) represents a non-invasive and practical method for assessing endogenous insulin production to facilitate diabetes classification. However, no study to date has reported the use of UCPCR in identifying LADA.

Patients and methods: A total of 574 subjects were included in our study (42 LADA, 61 T1DM, 471 T2DM). All participants were evaluated for UCPCR and underwent clinical and laboratory evaluations. UCPCR was compared among different subtypes of diabetes using multinomial regression analysis, and a receiver operating characteristic (ROC) curve was used to identify its performance in diagnosing LADA.

Results: UCPCR was lower in LADA $(0.4 \pm 0.6 \mathrm{nmol} / \mathrm{mmol})$ compared with T2DM $(1.2 \pm 0.9$ $\mathrm{nmol} / \mathrm{mmol})$, but higher than in T1DM $(0.2 \pm 0.3 \mathrm{nmol} / \mathrm{mmol})(\mathrm{p}<0.05)$. The association between UCPCR and LADA remained significant after adjusting for gender, age, age at diagnosis, body mass index, high-density lipoprotein cholesterol, and triglyceride (OR, 95\% confidence interval $(\mathrm{CI}), 0.29(0.09,0.95))$. The ROC curve revealed an area under the curve of 0.835 (95\% CI $(0.742-0.928), \mathrm{p}<0.001)$. The cut-off point for UCPCR $\leq 0.46 \mathrm{nmol} / \mathrm{mmol}$ was $82.1 \%$ for sensitivity and $76.7 \%$ for specificity in the diagnosis of LADA.

Conclusion: UCPCR may represent a non-invasive, simple, and practical measurement of insulin secretion for early discrimination of LADA in routine clinical practice.

Keywords: autoimmune diabetes, urinary C-peptide, $\beta$-cell function, non-invasive measurement

\section{Introduction}

Latent autoimmune diabetes in adults (LADA) is a slowly progressing form of autoimmune diabetes with older age at onset compared with classical type 1 diabetes (T1DM) and also characterized by $\beta$-cell associated antigen positivity. ${ }^{1}$ Some studies have reported that the decreasing rate of islet $\beta$-cell function in LADA was highly heterogeneous, and was approximately three times higher than that in patients with type 2 diabetes mellitus (T2DM). ${ }^{2}$ It is typically challenging to distinguish LADA from T2DM because they share a similar initial clinical presentation; however, LADA requires earlier insulin treatment compared with T2DM. ${ }^{3}$ Early insulin therapy leads to better preservation of metabolic control 
compared with treatment using oral hypoglycemic agents (OHA) only. ${ }^{4}$ Early diagnosis of LADA would, therefore, enable clear anticipation of disease progression and $\beta$-cell loss, which would reduce treatment inertia in insulin use when it is needed.

Islet $\beta$-cell function evaluation is a major concern when establishing a diagnosis of LADA and initiating insulin therapy. ${ }^{5}$ Although the detection of autoantibody, glutamic acid decarboxylase antibody (GADA), and insulinomaassociated-2 autoantibodies (IA-2A) has been considered essential for the diagnosis of LADA, practical considerations have limited the widespread use of these measurements. Poor endogenous insulin secretion is typically the main symptom of LADA in clinical practice, and some previous studies have therefore adopted endogenous insulin production assessment in LADA diagnosis. ${ }^{6} \mathrm{C}$-peptide is a reliable marker of endogenous insulin production and can be used even when patients are receiving insulin treatment. Serum C-peptide measurement when fasting or after stimulation is commonly used for the assessment of endogenous insulin secretion. ${ }^{7}$ However, these tests are invasive, and practicalities of collection limit widespread use. The urine $\mathrm{C}$-peptide creatinine ratio (UCPCR) represents a non-invasive practical alternative, and the stability of C-peptide in urine even allows outpatient or community testing. ${ }^{8}$ UCPCR had demonstrated high correlation with the "gold standard" measure of endogenous insulin secretion, the formal mixed-meal tolerance test (MMTT), in both T1DM and T2DM. ${ }^{8,9}$ Some studies have also supported the use of this novel, noninvasive marker for endogenous insulin secretion in the differential diagnosis of $\mathrm{T}_{1 \mathrm{DM}}{ }^{10}$ and maturity-onset diabetes of the young (MODY), ${ }^{11}$ and for detecting absolute insulin deficiency in T2DM. ${ }^{12}$ To the best of our knowledge, no studies to date have used UCPCR to differentiate LADA from other types of diabetes. The aim of the present study was therefore to evaluate the performance of UCPCR in establishing the diagnosis of LADA.

\section{Materials and Methods}

\section{Study Population}

A total of 574 participants with diabetes (61 T1DM, 471 T2DM, and 42 LADA) were recruited consecutively from 01 December 2017 to 01 March 2019 at the Endocrinology Department of Peking University People's Hospital. Inclusion criteria were age 18-80 years, and a clear diabetes classification diagnosis. Participants who were pregnant or experiencing a menstrual period at the time of urine sample collection were excluded from the study.

Diabetes was diagnosed in accordance with 1999 World Health Organization (WHO) criteria. ${ }^{13}$ Individuals with LADA were recruited based on criteria used in previous studies: (1) positive for at least one autoantibody (GADA, IA-2A); (2) age $\geq 30$ years at onset of diabetes; and (3) at least 6 months of therapy without insulin. ${ }^{14,15}$ T1DM was diagnosed in patients who met at least one of the following criteria: diagnosed at aged 35 years or younger, diabetes ketone acidosis (DKA) at the onset of disease, deficient C-peptide level, and signs of repeated ketosis when insulin was discontinued during the first 6 months after diagnosis. For those with age at onset between 30 and 35 years, whether DKA co-existed upon diagnosis, C-peptide level and hypoglycemic medical treatment during the first 6 months after diagnosis were carefully reviewed for differentiation of LADA and T1DM. T2DM was diagnosed by gold standard OGTT (oral glucose tolerance test) in adult patients with nonketosis onset diabetes and negative GADA and IA-2A. All diagnoses were made by one endocrinologist and confirmed by another blinded endocrinologist.

The study was approved by the Ethics Committee on Human Research of Peking University People's Hospital, and was performed in accordance with the Declaration of Helsinki (2013 revision). All subjects provided written informed consent to participate.

\section{Sample Collection}

All participants underwent physical examinations including height and weight measurement. Body mass index (BMI) was calculated as weight $(\mathrm{kg})$ divided by squared height $(\mathrm{m})$. Blood samples were collected in the morning after 8-12 hrs of fasting. Triglycerides (TG), total cholesterol (TC), high-density lipoprotein cholesterol (HDL-C), low-density lipoprotein cholesterol (LDL-C), fasting plasma glucose (FPG), and fasting C-peptide were determined by an enzyme immunoassay method using a biochemical analyzer (7600-120; Hitachi, Tokyo, Japan). The level of hemoglobin A1c (HbA1c) in whole blood was measured by automated high-performance liquid chromatography (Primus Ultra 2, Trinity Biotech, Bray, Co. Wicklow, Ireland) according to a standard procedure. GADA and IA-2A were measured in all subjects for diagnosis purposes using a biotin-coupled sandwich ELISA (RSR Limited, Pentwyn, Cardiff, UK). Blood samples testing was performed within $6 \mathrm{hrs}$ of collection. 


\section{UCPCR Analysis}

Urine samples were collected 2 hrs after a standard breakfast in hospital, which contained an appropriate calorie content and was adjusted for the age and weight of the participant under light physical labor conditions. Urinary C-peptide was tested using the same procedure as for serum C-peptide. Urinary creatinine level was measured using an electrochemiluminescence immunoassay on a Roche Diagnostics Cobas e601 analyzer. The urinary C-peptide level was divided by the urinary creatinine level to calculate UCPCR (nmol/mmol). Urine samples were stored at $-80^{\circ} \mathrm{C}$ and tested within $72 \mathrm{hrs}$ of collection.

\section{Statistical Analysis}

Results are presented as the mean, unless otherwise stated. Characteristics of participants with T1DM, T2DM, and LADA were compared by chi-squared test for categorical data (sex and marriage status) and ANOVA for other comparisons between the three groups. To adjust for possible confounders, differences in clinical features and biomarkers between the three groups (compared with T2DM) were assessed using multinomial regression to determine odds ratios. All parameters with a statistical significance $<0.05$ in the chi-squared and ANOVA analyses were included in the multinomial regression model. A receiver operating characteristic (ROC) curve was used to identify the cut-off point of UCPCR that yielded the optimal sensitivity and specificity (maximizing Youden index) for distinguishing LADA from T2DM. Differences were considered significant at $\mathrm{p}<$ 0.05. Statistical software SPSS 23.0 was used for the statistical analysis. MedCalc V15.2 was used for comparing different ROC curves of serum C-peptide and UCPCR in distinguishing LADA and T2DM.

\section{Results}

\section{Cohort Characteristics}

Sociodemographic and clinical characteristics for all participants are presented in Table $1.42 .9 \%$ of participants in LADA were male, while $59.0 \%$ and $56.9 \%$ of participants in T1DM and T2DM were male. LADA participants were younger than those with T2DM but older than those with T1DM $(53.7 \pm 12.0$ years vs $56.2 \pm 13.4$ years vs $42.9 \pm 18.5$

Table I Sociodemographic and Clinical Characteristics of Participants

\begin{tabular}{|c|c|c|c|c|c|}
\hline & All $n=574$ & TIDM n = 6I & T2DM $n=47$ I & LADA $n=42$ & P Valuet \\
\hline Male, n (\%) & $322(56.1)$ & $36(59.0)$ & $268(56.9)$ & $18(42.9)$ & $<0.05$ \\
\hline \multicolumn{6}{|l|}{ Marriage status, n (\%) } \\
\hline In marriage/cohabitation & $50 \mathrm{I}(87.3)$ & $40(65.6)$ & $421(89.4)$ & $40(95.2)$ & $<0.05$ \\
\hline Unmarried/divorce/widowed & $73(12.7)$ & $21(34.4)$ & $50(10.6)$ & $2(4.8)$ & $<0.05$ \\
\hline Mean (SD) age, years & $54.6(14.5)$ & $42.9(18.5)$ & $56.2(13.4)$ & $53.7(12.0)$ & $<0.05$ \\
\hline Mean (SD) age at diagnosis, years & $42.8(12.7)$ & $31.8(17.2)$ & 44.1 (II.5) & $43.8(10.4)$ & $<0.05$ \\
\hline Mean (SD) diabetes duration, years & $11.9(8.6)$ & II.I (I0.3) & I2.I (8.4) & $9.9(7.2)$ & 0.21 \\
\hline Mean (SD) BMI, $\mathrm{kg} / \mathrm{m}^{2} \mathrm{a}$ & $26.3(4.4)$ & $22.0(2.8)$ & $27.2(4.1)$ & $23.0(3.8)$ & $<0.05$ \\
\hline Mean (SD) HbAlc, \% ${ }^{\mathrm{a}}$ & $9.3(2.0)$ & $9.5(2.2)$ & $9.2(2.0)$ & $9.9(1.9)$ & 0.07 \\
\hline Mean $(\mathrm{SD})$ total cholesterol, $\mathrm{mmol} / \mathrm{L}^{\mathrm{a}}$ & $4.4(1.1)$ & $4.4(1.0)$ & $4.4(1.1)$ & $4.5(1.4)$ & 0.77 \\
\hline Mean (SD) HDL-C, mmol/ $\mathrm{L}^{\mathrm{a}}$ & $1.0(0.3)$ & I.3 $(0.3)$ & $1.0(0.2)$ & I.3 (0.4) & $<0.05$ \\
\hline Mean (SD) LDL-C, mmol// ${ }^{\mathrm{a}}$ & $2.8(0.8)$ & $2.8(0.9)$ & $2.8(0.8)$ & $2.9(0.9)$ & 0.78 \\
\hline Mean $(\mathrm{SD})$ triglyceride, $\mathrm{mmol} / \mathrm{L}^{\mathrm{a}}$ & $1.8(1.6)$ & $1.2(2.0)$ & $2.0(1.5)$ & I.I (0.5) & $<0.05$ \\
\hline Mean (SD) fast plasma glucose, $\mathrm{mmol} / \mathrm{L}^{\mathrm{a}}$ & $7.9(3.3)$ & $10.1(4.9)$ & $7.5(2.9)$ & $9.2(4.0)$ & $<0.05$ \\
\hline Mean (SD) fast C-peptide, $\mathrm{ng} / \mathrm{mL}^{\mathrm{a}}$ & $1.9(1.7)$ & $0.2(0.5)$ & $2.3(1.7)$ & $0.6(0.7)$ & $<0.05$ \\
\hline Mean (SD) UCPCR, $\mathrm{nmol} / \mathrm{mmol}$ & $1.0(0.9)$ & $0.2(0.3)$ & $1.2(0.9)$ & $0.4(0.6)$ & $<0.05$ \\
\hline
\end{tabular}

Notes: ${ }^{2} 2$ were missing for BMl; 15 was missing for $\mathrm{HbAlc} 5$ was missing for total cholesterol, HDL, LDL; 6 were missing for triglyceride; 4 were missing for fast plasma glucose; 2 were missing for fast $C$-peptide. $\nmid P$ value from chi-squared and ANOVA test. $P<0.05$ was considered significant.

Abbreviations: TIDM, type I diabetes mellitus; T2DM, type 2 diabetes mellitus; LADA, latent autoimmune diabetes in adults; BMI, body mass index; HbAIc, glycated hemoglobin AIc; HDL-C, high-density lipoprotein cholesterol; LDL-C, low-density lipoprotein cholesterol; UCPR, urinary C-peptide creatinine ratio. 
years, $\mathrm{p}<0.05)$. Age at diagnosis was markedly older in LADA than T1DM $(43.8 \pm 10.4$ vs $31.8 \pm 17.2, \mathrm{p}<0.05)$. BMI was highest in T2DM and lowest in T1DM, with LADA in the middle $\left(27.2 \pm 4.1 \mathrm{~kg} / \mathrm{m}^{2}\right.$ vs $22.0 \pm 2.8 \mathrm{~kg} / \mathrm{m}^{2}$ vs $\left.23.0 \pm 3.8 \mathrm{~kg} / \mathrm{m}^{2}, \mathrm{p}<0.05\right)$. HbA1c level was similar among all three groups $(\mathrm{p}=0.07)$. In the lipid profile, LADA and T1DM showed higher HDL-C and lower triglyceride than T2DM $(1.3 \pm 0.4 \mathrm{mmol} / \mathrm{L}$ and $1.3 \pm 0.3 \mathrm{mmol} / \mathrm{L}$ vs $1.0 \pm 0.2 \mathrm{mmol} / \mathrm{L}, \mathrm{p}<0.05 ; 1.1 \pm 0.5 \mathrm{mmol} / \mathrm{L}$ and $1.2 \pm 2.0$ $\mathrm{mmol} / \mathrm{L}$ vs $2.0 \pm 1.5 \mathrm{mmol} / \mathrm{L}, \mathrm{p}<0.05)$. Fasting C-peptide was highest in T2DM and lowest in T1DM while LADA in the middle $(2.3 \pm 1.7 \mathrm{ng} / \mathrm{mL}$ vs $0.2 \pm 0.5 \mathrm{ng} / \mathrm{mL}$ vs $0.6 \pm 0.7$ $\mathrm{ng} / \mathrm{mL}, \mathrm{p}<0.05)$. In general, LADA showed overlapping clinical features with T1DM and T2DM.

\section{Comparison of UCPCR Among Different Types of Diabetes}

UCPCR was lower in LADA $(0.4 \pm 0.6 \mathrm{nmol} / \mathrm{mmol})$ compared with T2DM $(1.2 \pm 0.9 \mathrm{nmol} / \mathrm{mmol})$ but higher than $\operatorname{T1DM}(0.2 \pm 0.3 \mathrm{nmol} / \mathrm{mmol})(\mathrm{p}<0.05)$. After adjustment of confounders (gender, age, age at diagnosis, BMI, HDL-C, triglyceride) in the multinomial regression model, UCPCR was still associated with LADA diagnosis: OR (95\% confidence interval (CI)), $0.29(0.09,0.95)$ (Table 2). Other factors associated with LADA diagnosis were fasting plasma glucose and fasting C-peptide (OR $(95 \% \mathrm{CI}$ ), $1.28(1.09,1.50)$ and $0.30(0.11,0.82)$, respectively).

\section{Performance of UCPCR in Distinguishing LADA from T2DM}

UCPCR was able to distinguish LADA from T2DM. The highest Youden index for identifying LADA from T2DM was $0.46 \mathrm{nmol} / \mathrm{mmol}$, with $82.1 \%$ sensitivity and $76.7 \%$ specificity (AUC, $0.835,95 \%$ CI $(0.742-0.928), \mathrm{p}<0.001$, Figure 1).

Furthermore, the comparison of ROC curves for UCPCR and serum C-peptide in distinguishing LADA and T2DM showed no significant difference (difference in AUC between UCPCR and serum C-peptide was $0.0549, \mathrm{Z}$ statistic was 1.72 , and $\mathrm{P}=0.08)$.

\section{Discussion}

In the present study, we used a novel non-invasive urine test for UCPCR to identify LADA diagnosis. UCPCR level was highest in T2DM and lowest in T1DM with LADA in the middle, and UCPCR was associated with LADA even after adjustment for confounders in multinomial regression. ROC analysis confirmed UCPCR $\leq 0.46 \mathrm{nmol} / \mathrm{mmol}$ as a cut-off point for distinguishing LADA from T2DM. To the best of our knowledge, the present study is the first to adopt UCPCR for the diagnosis of LADA.

LADA displays overlapping characteristics with T1DM and T2DM. Given its latent and slow progression, it can be difficult to distinguish LADA from T2DM at the onset of the disease. However, autoimmune destruction persists during the course of LADA, meaning that insulin treatment is inevitable for disease management. ${ }^{3}$ The availability of treatment strategies for LADA is limited by insufficient information from randomized controlled trials. Some studies have indicated that patients with LADA should be started on insulin or an incretin-based therapy, ${ }^{16,17}$ while sulfonylurea treatment is discouraged as it has been associated with a more rapid loss of insulin secretory capacity compared with insulin. ${ }^{18}$

Table 2 Predictive Markers Associated with LADA Diagnosis

\begin{tabular}{|c|c|c|c|c|}
\hline & \multicolumn{2}{|c|}{ Type $\mathbf{I}^{\mathbf{a}}$} & \multicolumn{2}{|c|}{ LADA $^{a}$} \\
\hline & OR & $95 \% \mathrm{Cl}$ & OR & $95 \% \mathrm{Cl}$ \\
\hline Male & 1.99 & $0.58,6.81$ & 0.68 & $0.24,1.90$ \\
\hline Age, years & 0.96 & $0.90,1.03$ & 0.95 & $0.90,1.01$ \\
\hline Age at diagnosis, years & 0.97 & $0.90,1.05$ & 1.04 & $0.97,1.12$ \\
\hline $\mathrm{BMI}, \mathrm{kg} / \mathrm{m}^{2}$ & 0.66 & $0.54,0.8 \mathrm{I}$ & 0.86 & $0.73,1.02$ \\
\hline HDL, mmol/L & 0.51 & $0.05,5.02$ & 3.17 & $0.46,21.7 \mathrm{I}$ \\
\hline Triglyceride, $\mathrm{mmol} / \mathrm{L}$ & 1.12 & $0.86,1.47$ & 0.58 & $0.24,1.43$ \\
\hline Fast plasma glucose, $\mathrm{mmol} / \mathrm{L}$ & 1.42 & $1.19,1.70$ & 1.28 & $1.09,1.50$ \\
\hline Fast C-peptide, ng/mL & 0.05 & $0.01,0.24$ & 0.30 & $0.11,0.82$ \\
\hline UCPCR, $\mathrm{nmol} / \mathrm{mmol}$ & 0.15 & $0.03,0.92$ & 0.29 & $0.09,0.95$ \\
\hline
\end{tabular}

Notes: ${ }^{a}$ Multinomial regression model was used for adjusting confounders. T2DM was the referent outcome and TIDM and LADA were compared with T2DM. Abbreviations: TIDM, type I diabetes mellitus; T2DM, type 2 diabetes mellitus; LADA, latent autoimmune diabetes in adults; BMI, body mass index; HDL-C, high-density lipoprotein cholesterol; UCPR, urinary C-peptide creatinine ratio. 


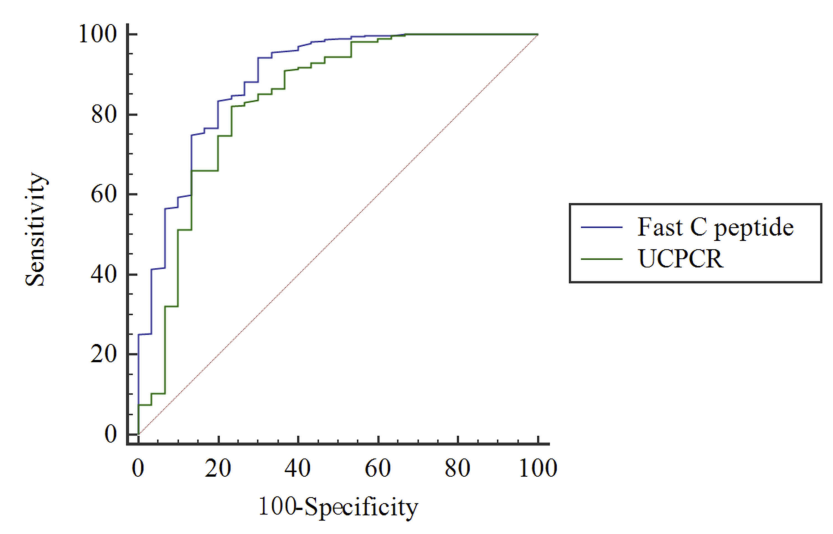

Figure I ROC curve to identify LADA from T2DM. The green curve represents a cutoff UCPCR $\geq 0.46 \mathrm{nmol} / \mathrm{mmol}$ for discriminating LADA from T2DM (AUC 0.835 ) with $82.1 \%$ sensitivity and $76.7 \%$ specificity. The blue curve represents the performance of fasting C-peptide in distinguishing between LADA and T2DM. The difference in AUC between UCPCR and serum C-peptide was 0.0549, Z statistic was 1.72 , and $P=0.08$. ROC, receiver operating characteristic; LADA, latent autoimmune diabetes in adults; T2DM, type 2 diabetes mellitus; UCPCR, urine $\mathrm{C}$-peptide creatinine ratio; AUC, area under the curve.

Personalized treatment is needed in LADA since the retrogression of endogenous insulin production is highly heterogeneous among patients with LADA. ${ }^{3}$ Furthermore, early detection of LADA, particularly as distinct from T2DM, is important so that insulin can be initiated earlier, thus facilitating a more rapid improvement in glycemic control as well as the preservation of residual $\beta$-cell function. ${ }^{3}$ Therefore, we focused on using a practical and easy urine test to differentiate LADA from T2DM in the current study.

Sociodemographic and clinical features of the participants in our study showed that LADA resembles the characteristics of both T1DM and T2DM in many ways, such as age at onset and BMI. Meanwhile, in our study, higher proportion of female patients was in LADA. This was in accordance with the Epidemiological Study of Risk Factors for LADA and Type 2 Diabetes (ESTRID) study, in which $45.7 \%$ and $39.2 \%$ of participants were female in LADA and T2DM, respectively. ${ }^{19}$ Another study in China focusing on pancreatic volume in LADA also suggested that the proportion of female participants was higher in LADA than in T2DM. ${ }^{20}$ This might be explained physiologically by the fact that LADA was associated with autoimmune destruction of $\beta$-cell, and autoimmunity diseases were more likely to happen in female.

In the present study, UCPCR was lower in LADA compared with T2DM but higher than in T1DM. Owing to the absolute insulin deficiency pathogenesis of T1DM, previous studies have also reported that UCPCR in T1DM is lower than in other subtypes of diabetes. ${ }^{21,22}$ Since
LADA resembles T1DM, but with a slower progression to absolute insulin requirement, it is reasonable to expect that UCPCR in LADA is higher than T1DM.

Diagnostic criteria remain controversial even more than 30 years after LADA was first described ${ }^{23}$ and commonly adopted items include the measurement of circulating islet autoantibodies, which is expensive and difficult to implement. Some researchers have adopted C-peptide in the diagnosis of LADA, ${ }^{24,25}$ as it can represent endogenous insulin production and facilitate treatment decisions. UCPCR is a new, noninvasive, and stable marker for endogenous insulin secretion, and has shown good correlation with serum C-peptide during a mixed-meal tolerance test. ${ }^{6,10}$ Some studies have started to use UCPCR as a convenient tool for evaluating islet $\beta$-cell function and even to facilitate screening for monogenic diabetes in young-onset patients. ${ }^{26,27}$ Sebahat and colleagues reported that UCPCR $\geq 0.22 \mathrm{nmol} / \mathrm{mmol}$ could distinguish MODY from T1DM with $96.3 \%$ sensitivity and $85.7 \%$ specificity. ${ }^{24}$ Shields and colleagues even used UCPCR $\geq 0.2$ $\mathrm{nmol} / \mathrm{mmol}$ as a cut-off point for screening for monogenic diabetes in a population-based cohort. ${ }^{22}$ However, no study to date has reported the possible role of UCPCR in identifying LADA. In the present study, UCPCR $\leq 0.46 \mathrm{nmol} / \mathrm{mmol}$ was established as a cut-off point for distinguishing LADA from T2DM, and the performance of UCPCR in identifying LADA was similar to that of fasting C-peptide.

UCPCR, therefore, represents a non-invasive and stable measurement alternative to traditional serum $\mathrm{C}$-peptide testing for detecting endogenous insulin production. UCPCR is capable of determining $\beta$-cell function in long-duration T1DM with minimal insulin secretion. ${ }^{28}$ Other studies have even expanded UCPCR use to an outpatient setting for identifying T2DM and MODY. ${ }^{11}$ The reliability and noninvasiveness of UCPCR may, therefore, be of benefit in other clinical settings.

The present study had some limitations, including its single-center design with a relatively small patient cohort. Further studies using UCPCR to identify LADA in a larger, newly diagnosed population would extend the validity of our findings.

The gold-standard diagnosis criteria for LADA remain positive for at least one autoantibody (GADA, IA-2A), age at onset of diabetes $\geq 30$ years old, and at least 6 months without insulin treatment after diagnosis. Considering the practical limitations of this criteria in the general population, the UCPCR cut-off can serve as an initial screening measure for LADA. Similarly, the UCPCR cut-off can be 
used to differentiate LADA from T2DM as the LADA patients may need early insulin administration.

\section{Conclusion}

Frequent misdiagnosis as T2DM means that patients with LADA are typically treated with therapies indicated for non-autoimmune forms of diabetes, resulting in a rapid decline of $\beta$-cell function, particularly in younger and leaner subjects and those with high GADA titers. ${ }^{29,30}$ Thus, early detection of LADA, especially to distinguish LADA from T2DM, is critical for appropriate treatment selection. Our study describes a non-invasive and convenient method for identifying LADA which may contribute to the early detection of this unique subtype of diabetes and facilitate personalized treatment strategies.

\section{Acknowledgments}

We are grateful to the patients, nurses, and physicians who participated in this study. The authors would also like to thank Dr. Chong Liu from Peking University for his kind support with data analysis.

\section{Funding}

This study was supported by National Natural Science Foundation of China (81700722) and the National Key Research and Development Program (No. 2016YFC1304901).

\section{Disclosure}

The authors report no conflicts of interest in this work.

\section{References}

1. Buzzetti R, Di Pietro S, Giaccari A, et al.; Non Insulin Requiring Autoimmune Diabetes Study Group. High titer of autoantibodies to GAD identifies a specific phenotype of adult-onset autoimmune diabetes. Diabetes Care. 2007;30(4):932-938. doi:10.2337/dc06-1696

2. Yang L, Zhou ZG, Huang G, et al. Six-year follow-up of pancreatic beta cell function in adults with latent autoimmune diabetes. World J Gastroenterol. 2005;11(19):2900-2905. doi:10.3748/wjg.v11.i19.2900

3. Andersen CD, Bennet L, Nyström L, et al. Worse glycaemic control in LADA patients than in those with type 2 diabetes, despite a longer time on insulin therapy. Diabetologia. 2013;56(2):252-258. doi:10.1007/s00125-012-2759-y

4. Thunander $\mathrm{M}$, Thorgeirsson $\mathrm{H}$, Törn $\mathrm{C}$, et al. $\beta$-cell function and metabolic control in latent autoimmune diabetes in adults with early insulin versus conventional treatment: a 3-year follow-up. Eur J Endocrinol. 2011;164(2):239-245. doi:10.1530/EJE-10-0901

5. Buzzetti R, Zampetti S, Maddaloni E. Adult-onset autoimmune diabetes: current knowledge and implications for management. Nat Rev Endocrinol. 2017;13(11):674-686. doi:10.1038/nrendo.2017.99

6. Mishra R, Hodge KM, Cousminer DL, et al. A global perspective of latent autoimmune diabetes in adults. Trends Endocrinol Metab. 2018;29(9):638-650. doi:10.1016/j.tem.2018.07.001
7. Jones AG, Hattersley AT. The clinical utility of C-peptide measurement in the care of patients with diabetes. Diabet Med. 2013;30 (7):803-817. doi:10.1111/dme.12159

8. McDonald TJ, Knight BA, Shields BM, et al. Stability and reproducibility of a single-sample urinary C-peptide/creatinine ratio and its correlation with 24-h urinary C-peptide. Clin Chem. 2009;55 (11):2035-2039. doi:10.1373/clinchem.2009.129312

9. Palmer JP, Fleming GA, Greenbaum CJ, et al. C-peptide is the appropriate outcome measure for type 1 diabetes clinical trials to preserve beta-cell function: report of an ADA workshop, 21-22 October 2001. Diabetes. 2004;53(1):250-264. doi:10.2337/diabetes.53.1.250

10. Besser RE, Ludvigsson J, Jones AG, et al. Urine C-peptide creatinine ratio is a noninvasive alternative to the mixed-meal tolerance test in children and adults with type 1 diabetes. Diabetes Care. 2011;34 (3):607-609. doi:10.2337/dc10-2114

11. Besser RE, Shields BM, Hammersley SE, et al. Home urine C-peptide creatinine ratio (UCPCR) testing can identify type 2 and MODY in pediatric diabetes. Pediatr Diabetes. 2013;14(3):181-188. doi:10.1111/pedi. 12008

12. Hope SV, Jones AG, Goodchild E, et al. Urinary C-peptide creatinine ratio detects absolute insulin deficiency in type 2 diabetes. Diabet Med. 2013;30(11):1342-1348. doi:10.1111/dme.12222

13. Alberti KG, Zimmet PZ. Definition, diagnosis and classification of diabetes mellitus and its complications. Part 1: diagnosis and classification of diabetes mellitus provisional report of a WHO consultation. Diabet Med. 1998;15(7):539-553. doi:10.1002/(ISSN)1096-9136

14. Mishra R, Chesi A, Cousminer DL, et al. Relative contribution of type 1 and type 2 diabetes loci to the genetic etiology of adult-onset, non-insulin-requiring autoimmune diabetes. BMC Med. 2017;15 (1):88. doi:10.1186/s12916-017-0846-0

15. Deng C, Xiang Y, Tan T, et al. Altered peripheral B-lymphocyte subsets in type 1 diabetes and latent autoimmune diabetes in adults. Diabetes Care. 2016;39(3):434-440. doi:10.2337/dc15-1765

16. Johansen OE, Boehm BO, Grill V, et al. C-peptide levels in latent autoimmune diabetes in adults treated with linagliptin versus glimepiride: exploratory results from a 2-year double-blind, randomized, controlled study. Diabetes Care. 2014;37(1):e11-e12. doi:10.2337/dc13-1523

17. Buzzetti R, Pozzilli P, Frederich R, et al. Saxagliptin improves glycaemic control and C-peptide secretion in latent autoimmune diabetes in adults (LADA). Diabetes Metab Res Rev. 2016;32 (3):289-296. doi:10.1002/dmrr.2717

18. Brophy S, Davies H, Mannan S, et al. Interventions for latent autoimmune diabetes (LADA) in adults. Cochrane Database Syst Rev. 2011;9:CD006165. doi:10.1002/14651858.CD006165.pub3

19. Hjort R, Ahlqvist E, Carlsson PO, et al. Overweight, obesity and the risk of LADA: results from a Swedish case-control study and the Norwegian HUNT Study. Diabetologia. 2018;61(6):1333-1343. doi:10.1007/s00125-018-4596-0

20. Lu J, Hou X, Pang C, et al. Pancreatic volume is reduced in patients with latent autoimmune diabetes in adults. Diabetes Metab Res Rev. 2016;32(8):858-866. doi:10.1002/dmrr.2806

21. Besser RE, Shepherd MH, McDonald TJ, et al. Urinary C-peptide creatinine ratio is a practical outpatient tool for identifying hepatocyte nuclear factor 1-\{alpha\}/hepatocyte nuclear factor 4- $\alpha$ maturityonset diabetes of the young from long-duration type 1 diabetes. Diabetes Care. 2011;34(2):286-291. doi:10.2337/dc10-1293

22. Yılmaz Agladioglu S, Sagsak E, Aycan Z. urinary c-peptide/creatinine ratio can distinguish maturity-onset diabetes of the young from type 1 diabetes in children and adolescents: a single-center experience. Horm Res Paediatr. 2015;84(1):54-61. doi:10.1159/000375410

23. Liao Y, Xiang Y, Zhou Z. Diagnostic criteria of latent autoimmune diabetes in adults (LADA): a review and reflection. Front Med. 2012;6(3):243-247. doi:10.1007/s11684-012-0201-y

24. Weber P, Ambrosova P, Canov P, et al. GAD antibodies in T1D and LADA-relations to age, BMI, c-peptide, IA- 2 and HLA-DRB $1 * 03$ and DRB1*04 alleles. Adv Gerontol. 2011;24(2):312-318. 
25. Weber $\mathrm{P}$, Meluzínová $\mathrm{H}$, Kubesová $\mathrm{H}$, et al. Type 1 diabetes and LADA - occurrence of HLA-DRB $1 * 03$ and DRB $1 * 04$ alleles in two age different groups of diabetics. Adv Gerontol. 2009;23(2):243-248.

26. Shields BM, McDonald TJ, Oram R, et al.; TIGI Consortium. C-Peptide decline in type 1 diabetes has two phases: an initial exponential fall and a subsequent stable phase. Diabetes Care. 2018;41(7):1486-1492. doi:10.2337/dc18-0465

27. Shields BM, Shepherd M, Hudson M, et al.; UNITED study team. Population-based assessment of a biomarker-based screening pathway to aid diagnosis of monogenic diabetes in young-onset patients. Diabetes Care. 2017;40(8):1017-1025. doi:10.2337/ dc17-0224
28. Oram RA, Jones AG, Besser RE, et al. The majority of patients with long-duration type 1 diabetes are insulin microsecretors and have functioning beta cells. Diabetologia. 2014;57(1):187-191. doi:10.1007/s00125-013-3067-x

29. Maddaloni E, Lessan N, Al Tikriti A, et al. Latent autoimmune diabetes in adults in the United Arab Emirates: clinical features and factors related to insulin-requirement. PLoS One. 2015;10(8): e0131837. doi:10.1371/journal.pone.0131837

30. Zampetti S, Campagna G, Tiberti C, et al. High GADA titer increases the risk of insulin requirement in LADA patients: a 7-year follow-up (NIRAD study 7). Eur J Endocrinol. 2014;171(6):697-704. doi:10.1530/EJE-140342

Diabetes, Metabolic Syndrome and Obesity: Targets and Therapy

\section{Publish your work in this journal}

Diabetes, Metabolic Syndrome and Obesity: Targets and Therapy is an international, peer-reviewed open-access journal committed to the rapid publication of the latest laboratory and clinical findings in the fields of diabetes, metabolic syndrome and obesity research. Original research, review, case reports, hypothesis formation, expert opinion and commentaries are all considered for publication. The manuscript management system is completely online and includes a very quick and fair peer-review system, which is all easy to use. Visit $\mathrm{http}: / / \mathrm{www}$.dovepress.com/testimonials.php to read real quotes from published authors. 